\title{
La idea de Universidad en el papa Francisco
}

\author{
JORGE BAEZA CORREA* \\ Universidad Católica Silva Henríquez (Chile) \\ jbaeza@ucsh.cl
}

\begin{abstract}
Resumen
La idea de la Universidad en el papa Francisco, expresada en sus diversos discursos, cartas y homilías dirigidas al mundo universitario, permiten identificar un conjunto de desafíos que invitan a volver a visitar Ex Corde Ecclesiae ya 30 años después de su publicación. Este artículo, a través de un análisis documental de 30 textos del papa Francisco, específicamente referidos a la universidad o estudiantes universitarios, mediante un análisis estadístico de datos textuales y luego un análisis cualitativo de carácter temático, permiten concluir que para el papa Francisco, utilizando sus propias expresiones: La realidad mundial es una "cultura de descarte", que puede ser superada por una "cultura de encuentro", que requiere que la universidad se convierta en una universidad "en salida". Del análisis se aprecia que el papa Francisco, centra sus textos sobre las universidades, principalmente, en el quehacer de ellas en la sociedad actual, reconceptualizando con estos énfasis en el hacer, el ser mismo de la universidad.
\end{abstract}

Palabras claves: Francisco; universidad; estudiante universitario; universidad católica.

\section{The idea of University in pope Francis}

\begin{abstract}
The idea of the University in pope Francis, expressed in its various speeches, letters and homilies addressed to the university world, identifies a set of challenges that invite you to revisit Ex Corde Ecclesiae 30 years after its publication. This article, through a documentary analysis of 30 texts by pope Francis, which refer specifically to the university or university students, through a statistical analysis of textual data and then a thematic analysis, allows to conclude that for pope Francis, using his own expressions: World reality is a "culture of discard", which can be overcome by a "culture of encounter", which requires the university to become a "come out" university. It is clear from the analysis that Pope Francis, focuses his texts on universities, mainly, on the work of them in today's society, reconceptualizing with these emphases, the very being of the university.
\end{abstract}

Key words: Francisco; university; university student; catholic university.

* Sociólogo. Doctor en Ciencias de la Educación. Actualmente Investigador del Centro de Investigación en Ciencias Sociales y Juventud de la Universidad Católica Silva Henríquez. Durante el período noviembre 2011 - diciembre 2017 fue Rector de dicha Casa de Estudios Superiores. Integra el Comité Científico de la Fundación Pontificia Gravissimum Educationis. Ha publicado recientemente Young People's Reality. Educational Challenges (2020, junto a Rosa Aparicio). 


\section{INTRODUCCIÓN}

A treinta años de la promulgación de Ex Corde Ecclesiae (1990) y en los días que el papa Francisco ha hablado de la "Universidad del Sentido", en su video mensaje con ocasión del ciber-encuentro mundial organizado por la Fundación Scholas occurrentes (junio 2020); bien vale detenerse en identificar cuáles son las ideas principales de Francisco sobre Universidad.

Sin duda alguna, todas y cada una de las palabras de Ex Corde Ecclesiae tienen en sus aspectos más sustantivos una vigencia plena, pero no podemos dejar de reconocer que, en 30 años, muchos de los desafíos más inmediatos del quehacer universitario han experimentado modificaciones importantes. Treinta años en el mundo universitario no es un tiempo menor, los jóvenes que están hoy en los primeros años universitarios, nacieron hace 18, 19 o 20 años.

Recordando el conocido trabajo de Baber, Donnelly \& Rizvi (2013: 4), no se puede dejar de parafrasear el título de dicho informe: el futuro se avalancha sobre las universidades. Tal como sostiene el informe: "Los modelos de educación superior que marcharon triunfalmente en todo el mundo en la segunda mitad del siglo XX están rotos (...), en los próximos 20 años las universidades se enfrentan a la transformación".

Sin ser exhaustivo, hoy es fácil identificar que son muchos los desafíos que enfrentan las universidades en todos sus ámbitos. El docente actual se enfrenta a un sujeto muy distinto a él cuando él fue estudiante; se experimenta un crecimiento vertiginoso de la población universitaria, donde si bien, todavía hay una amplia brecha en el acceso entre los diferentes grupos sociales, no se puede dejar de reconocer que, en la actualidad, hay en las aulas una composición social diferente a la de muy pocas décadas atrás. La educación superior se ha masificado y los niveles de selectividad, con una oferta muy diferenciada, son más fácil de superar. La equidad en la educación superior, cada vez más, ya no es tanto un tema de cobertura, sino una tarea de pertinencia, de acoger adecuadamente a quienes vienen llegando. Además, el trabajo docente de hoy debe estar más centrado en aprender a aprender, que en el aprender conocimientos que prontamente serán obsoletos. Es también una realidad de profundo individualismo y competitividad, que requiere formar para el trabajo en equipo y el aprendizaje colaborativo.

Probablemente nunca antes la educación superior había tenido disponible tal nivel y cantidad de avances tecnológicos que pueden ser utilizados en sus aulas y ello ha quedado, visiblemente demostrado en este tiempo de pandemia. Los estudiantes actuales pueden con sus teléfonos celulares, asistir a clases a miles de kilómetros y la posibilidad de apren- 
der con ellos en todas partes y en todo momento, relativizando en gran medida el tiempo y el espacio. La realidad aumentada, posibilita a profesores y estudiantes un acercamiento no invasivo a realidades sensibles y el uso del juego (la gamificación), se populariza como un mecanismo de enseñanza. La posibilidad de reunir a expertos y estudiantes de todo el mundo en tele conferencias y la incorporación de los MOOC (cursos online masivos y abiertos) están ayudando a la circulación libre del conocimiento; pero, también, abren nuevas brechas con quienes no tienen la alfabetización digital requerida y el manejo del inglés, como lengua universal.

Son numerosos los informes que sostienen que los trabajos del futuro que fusionan lo físico, lo digital y lo biológico, no encuentran el espacio necesario en el medio universitario. En términos contrarios, en la actualidad se estaría impartiendo formación en las aulas, para trabajos que ya no existirán en un futuro. Este es un dato que está llamando a la necesidad de una mayor y más profunda vinculación entre las universidades y el mundo laboral. Más aún, cuando la necesidad de un aprendizaje a lo largo de la vida, está desdibujando los contornos de diferenciación entre los centros de Educación Superior y otras instituciones o experiencias que forman a las personas en la práctica. Se impone con fuerza la necesidad de reconocer, que las universidades están perdiendo el monopolio de la formación y con ello de la acreditación de competencias. Por otro lado, los centros formativos tendrán que prepararse para una sociedad en constante evolución y cada vez más automatizada, pero que, a su vez, exigen una formación más humanista para desarrollar capacidades para la flexibilidad y la adaptación intelectual (resiliencia), dado los continuos cambios ya no solo laborales, sino que también de profesión; como también, de espacios territoriales dada la internacionalización.

Por último, tampoco se puede dejar de reconocer, que el futuro encierra nuevos y desconocidos retos que hacen necesario un cambio en la organización y gobierno de las instituciones universitarias. Desde luego, una organización más flexible y más ágil, tarea extremadamente difícil de realizar, en una organización que construye su identidad en el apego a las tradiciones. Se requiere avanzar a una institución que construya su identidad valorando el pasado, reconociendo las exigencias del presente, pero proyectándose al futuro con que sueña. Una universidad, con menos departamentos disciplinarios y más trabajo colaborativo entre grupos académicos distintos; con el otorgamiento de micro títulos que se pueden ir ampliando en el transcurso de la vida; con espacios mayormente para el trabajo colaborativo y cada vez menos presencial, una organización más de construcción de alianza que de disputa de espacio (de asociación a otras instituciones y de economía de escala). En un marco de 
cada vez mayor exigencia por calidad, el financiamiento de la educación superior no podrá estar basado tanto en la incorporación de nuevos recursos, sino que, en la creación de nuevos productos, lo que debería significar, que parte importante de la gestión directiva estará más concentrada en incentivar la creatividad y la innovación al interior de la institución, que en atraer recursos desde afuera.

Todavía algo más, así como los hombres y mujeres de hoy parecen haber renunciado al intento de cambiar el mundo, para buscar el propio cambio y adecuarse al mundo; como tampoco, ya no aspiran a convertirse en un nuevo ser, en una sociedad de iguales, sino que ambicionan y compiten por ser "ganadores". Lo mismo pareciera ser que acontece con algunas universidades, renuncian a su función crítica y de compromiso con la cultura de su nación, preocupadas de ser ellas vencedoras en un medio cada vez más competitivo y de financiamiento privatizado; como también, son más descomprometidas con sus lugares de quehacer, producto de la existencia de transnacionales universitarias.

Toda esta realidad de cambios profundos y nuevos desafíos, ha llevado a que el Informe del Global University Network for Innovation: Higher Education in the World 7 (GUNI, 2019: 546), que tiene como objetivo proporcionar a la comunidad académica un diagnóstico y análisis de la situación actual en el mundo universitario, a sostener que: "Las sociedades están asistiendo a cambios profundos con claras implicaciones para el futuro; estas transformaciones ambientales, científicas, tecnológicas, culturales y sociales están presentando desafíos trascendentales en términos de pensamiento y replanteamiento del significado y valor de la experiencia humana, e incluso de lo que significa ser humano".

En síntesis, no hay dudas, en estos últimos 30 años las Universidades están enfrentando nuevos desafíos, ello además es lo que reconoce Monseñor Zani (2015: 1), Obispo Secretario de la Congregación para la Educación al conmemorase los 25 años de Ex Corde Ecclesiae: "Hoy, los sistemas universitarios deben enfrentarse con nuevos desafíos y con riesgos inéditos que los interrogan no en modo superficial y extrínseco, sino que los obligan a repensar la propia naturaleza y la propia finalidad".

El tema universitario ha sido siempre un tema querido y cercano a los últimos Pontífices. Para Juan Pablo II y Benedicto XVI, constituyó siempre parte importante de sus preocupaciones, seguramente, por sus experiencias universitarias y su vocación académica; de aquí sus innumerables textos sobre la materia y la conformación de una idea de universi- 
dad, claramente marcada con un sello propio ${ }^{1}$. En esta misma línea y habiendo establecido que son numerosos e importantes los nuevos desafíos al mundo universitario, resulta valido preguntarse: ¿cuáles son los acentos que el papa Francisco coloca como preocupación principal al hablar a las universidades y a los universitarios? Ello es lo que intentaremos responder en las siguientes páginas, adoptando una metodología que permita por sobre todo escuchar al Santo Padre, al sistematizar sus cartas, saludos, homilías y escritos sobre la materia.

\section{Metodología}

La metodología que se ha optado, es de un acercamiento progresivo a la temática. En un primer momento, algo que es cada vez más frecuente en el análisis documental: un análisis estadístico de datos textuales. Lo que a juicio de Páramo (2010: 142), implica "contar las ocurrencias de las unidades verbales básicas (...) se analizan las listas de frecuencias de palabras (...) luego se deben tomar aquellas con mayor frecuencia y que son significativas a los fines del estudio". En este caso para un primer acercamiento, mediante el uso del programa de acceso libre "Nube de Palabras" (www.nubedepalabras.es), se cuentan palabras para determinar el número de veces utilizadas y se elaboran gráficas, que da cuenta visualmente de la frecuencia de uso de dichas palabras.

Realizado lo anterior, se ingresa al segundo método de trabajo que corresponde a lo llamado análisis temático, una variante del análisis de discurso, que tiene la intención de identificar los elementos comunes en los textos. El análisis temático, sostienen Rodríguez y Garrigós, "clasifica el corpus de textos a partir de la delimitación del conjunto de temas representativos del contenido de los textos y pertinente a los fines de la investigación. En este sentido, el análisis temático desarrolla el análisis del discurso en su nivel informacional". Es un método, añaden, que "para el análisis de datos textuales se inicia con la pregunta ¿`sobre qué versa esto?; que continua con el listado de todos los temas identificados" (2017: 184).

Este método, como señalan Braun \& Clarke (2006: 6), "permite identificar, analizar y reportar patrones (temas) dentro de datos" ${ }^{2}$. Un

1 Solo a modo de referencia, en acceso libre en internet, se puede encontrar para cada uno de los Pontífices un trabajo específico referido al tema: Cuartas (2003) y Viñado (2017).

2 Si bien se utiliza el análisis temático como un método principal, no hay un uso detallado de cada una de sus partes. Licencia que las mismas autoras animan, invitando a un uso flexible de este método. Es importante tener presente al respecto, que lo principal siempre es el objetivo, ya que el objetivo es el que ordena el trabajo de 
tema, para Braun \& Clarke (2006: 10), es aquel que "captura algo importante sobre los datos en relación con la pregunta de investigación, y representa algún nivel de respuesta estructurada o con significado dentro del conjunto de datos". Rodríguez y Garrigós (2017: 185), agregan a ello, que, en la identificación de los temas, los investigadores se centran "en la recurrencia, la repetición y contundencia de los mismos".

En este caso, el trabajo se inicia teniendo como base la página oficial del Vaticano (http://www.vatican.va/content/vatican/es.html), la cual reúne la totalidad de los textos firmados por el papa Francisco. De esa producción se consideraron, desde el inicio de su pontificado, todos aquellos textos que tienen como destinatarios una universidad en particular o un grupo de universitarios. El criterio de selección, por lo tanto, es a quién o quiénes está dirigido; por lo cual no se consideraron aquellos textos donde hay referencias a la universidad o universitarios, pero no están dirigidos específicamente a dichos destinatarios.

En una primera revisión, se encontraron en función del destinario explícito en el título del escrito o intervención transcrita, un total de 33 textos, los que revisados en sus contenidos disminuyeron a 30 al descartar a aquellos que son saludos formales a universidades o cartas dirigidas a universidades a nombre del Santo Padre, pero firmadas por otra persona, como es el caso del Secretario de Estado. Los 30 textos considerados, incluyen discursos, homilías, mensajes, cartas, saludos y video-mensajes, según la propia clasificación que presenta el texto. Textos que, en su mayoría, están en español en la página antes citada y solo un mínimo debieron ser traducidos (alemán o italiano), lo que permitió trabajar mayormente $(88,0 \%)$ con textos traducidos en forma oficial.

\section{RESULTADOS}

El primer acercamiento al conjunto de textos, como ya se mencionó en la metodología, lo constituye un trabajo cuantitativo, que implicó la construcción de un solo corpus en función de los 30 textos considerados, lo que permitió identificar palabras y contar el número de veces utilizadas. En este primer proceso, como aconseja la metodología, primero se retiraron la mayor parte posible de palabras que operan como conectores (por ejemplo: $y$, de, o, ...) y luego, se unieron aquellas que son conjugaciones de una misma palabra o presentan variaciones por estar

investigación. Como sostiene Santander (2011: 215): “el análisis es muy dependiente de nuestro objetivo general o de nuestra hipótesis. Al estar orientado a probar la hipótesis o a cumplir el objetivo general, el tipo de análisis también puede sufrir fuertes variaciones de caso en caso". 
escritas como mayúscula o minúscula (por ejemplo: universidad, Universidad o universidades). Este primer trabajo, permite ya un primer acercamiento posible a través de la construcción de una nube de palabras, donde se destacan con nitidez aquellas de mayor uso.

\section{Grafico $\mathrm{N}^{\circ} 1$ Nube de palabras, total del corpus}

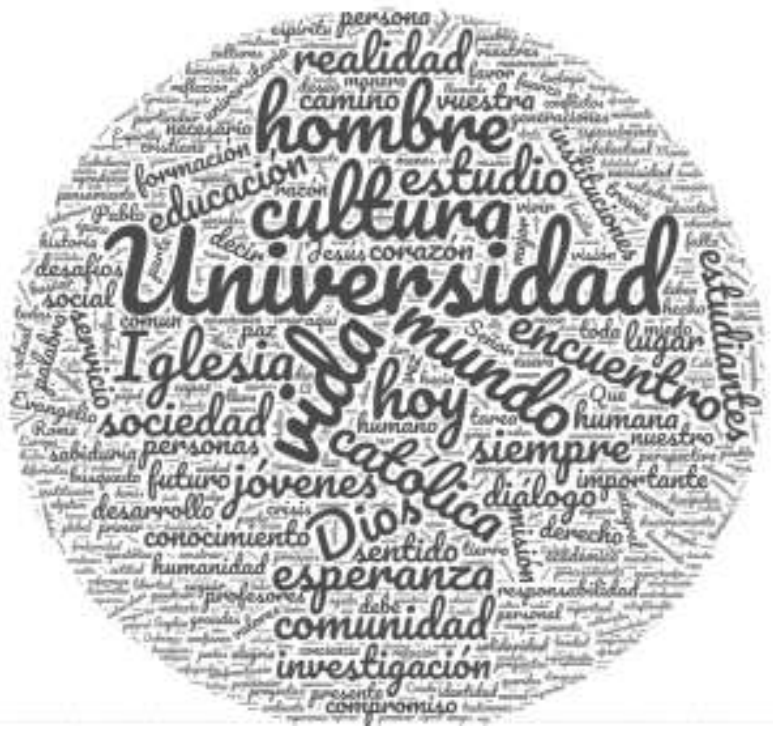

Luego, en un segundo momento, dentro de esta primera etapa de acercamiento, se adoptó la decisión de establecer un punto de corte, que permitiera trabajar con un número acotado de palabras, desde luego, las mayormente citadas. En este caso se colocó como parámetro un número igual o superior a 30 menciones, con lo cual se establece un total a considerar de 20 palabras.

Tabla $\mathrm{N}^{\circ}$ 1: Número de veces citada cada palabra

\begin{tabular}{|c|c|c|c|c|}
\hline 123 & 86 & 69 & 65 & 59 \\
\hline universidad & vida & cultura & hombre & mundo \\
\hline 53 & 49 & 49 & 46 & 45 \\
\hline hoy & iglesia & católica & Dios & encuentro \\
\hline 45 & 43 & 40 & 39 & 39 \\
\hline estudio & esperanza & realidad & jóvenes & comunidad \\
\hline 39 & 39 & 35 & 34 & 33 \\
\hline sociedad & siempre & educación & estudiantes & investigación \\
\hline
\end{tabular}


Sin ingresar a un análisis detallado de todas y cada una de las palabras, no deja de ser interesante apreciar, que después de la palabra universidad, que resulta lógico que se ubique en primer lugar de citaciones, la segunda sea la palabra "vida". La palabra vida se presenta mayormente unida a otras palabras, generando expresiones tales como: santidad de vida; vida cristiana; vida nueva; vida interior; dar vida; sentido de la vida y programa de vida. Hay en este conjunto de expresiones tomadas como una totalidad, un doble llamado: tanto hacia una interioridad como, a su vez, una invitación hacia el "exterior", un compromiso con los demás, como se aprecia en la siguiente frase: "La investigación y el estudio se deben integrar con la vida personal y comunitaria, con el compromiso misionero, con la caridad fraterna y el gesto de compartir con los pobres, con la atención a la vida interior en la relación con el Señor" (Francisco, 2014c).

No obstante, lo señalado, al recorrer los textos del papa Francisco se encuentra, que, sin dejar nunca de hacer alusión a esta doble tarea interna-externa, hay en sus textos un continuo relevar en un grado importante el compromiso de vida con los demás: "Por favor, no miréis la vida desde el balcón. Implicaos allí donde están los desafíos, que os piden ayuda para llevar adelante la vida, el desarrollo, la lucha en favor de la dignidad de las personas, la lucha contra la pobreza, la lucha por los valores y tantas luchas que encontramos cada día" (Francisco, 2013b). Es claro en el Santo Padre, viendo el listado de las palabras más utilizados, que el tema del mundo, del hoy, la realidad y la sociedad son temas de su preocupación.

Una segunda palabra que llama la atención en este listado, es "siempre". Siempre, es una expresión de certeza, pero también de imperativo. La RAE indica que siempre "es en todo o cualquier tiempo". El papa Francisco la utiliza como certeza en un mundo en cambio y como imperativo, en cuanto a conducta a seguir:

Está siempre con nosotros. Se coloca, con respeto y discreción, a lo largo del camino de nuestra vida, nos sostiene especialmente en la hora de la pérdida y la derrota, en el momento de la debilidad y del pecado, para volvernos a situar siempre en el camino (Francisco, 2017b).

La Universidad, enfocada en su misión, debería estar abierta siempre a crear un archipiélago capaz de interconectar lo que social y culturalmente puede llegar a concebirse como separado (Francisco, 2019g).

Hay en estas dos palabras, "vida" y "siempre", un aspecto común, un doble movimiento, hacia lo interno y hacia lo exterior, a la certeza que 
afianza y al impulso que moviliza, algo que está en varios otros aspectos del discurso del papa Francisco. Por ejemplo, cuando sostiene en la Exhortación Apostólica Gaudete et Exsultate (2018: N²6)": "No es sano amar el silencio y rehuir el encuentro con el otro, desear el descanso y rechazar la actividad, buscar la oración y menospreciar el servicio".

El listado de palabras más citadas, ofrece también una posibilidad de hipotetizar respecto a la forma en que el papa Francisco articula su discurso con relación a universidad, lo que desde luego habrá que comprobar con el análisis siguiente. En este listado (así se buscó) hay un interlocutor al cual se le habla: la universidad, la comunidad académica y los estudiantes y por extensión al hombre en general y a los jóvenes en particular. Como hipótesis, al revisar el listado de palabras más citadas, al parecer hay aspectos sobre los cuáles se habla: la vida, el mundo, hoy, la realidad y la sociedad; como también, sobre aspectos más específico del mundo universitario: la cultura, el estudio, la educación y la investigación. Hay también lugares desde dónde se habla: Dios, la iglesia y la catolicidad y existe, además, dos acentos particulares con qué se habla: la esperanza y el valor del encuentro.

\section{La realidad}

Un primer aspecto que se encuentra al hacer un análisis temático de los textos del papa Francisco dirigidos al mundo universitario, es la identificación de un eje referido al diagnóstico de la realidad. Un eje temático que articula a su alrededor temas como la realidad social, la situación del mundo, lo que se vive actualmente y lo que acontece hoy.

Para el papa Francisco, un aspecto sustantivo que caracteriza el momento actual es la realidad de un profundo, vertiginoso y amplísimo cambio.

Cierto, cada época de la historia lleva en sí elementos críticos, pero, al menos en los últimos cuatro siglos, no se han visto tan sacudidas las certezas fundamentales que constituyen la vida de los seres humanos como en nuestra época. Pienso en el deterioro del medio ambiente: esto es peligroso, pensemos un poco adelante, en la guerra del agua, que viene; en los desequilibrios sociales; en el terrible poder de las armas (...); en el sistema económico-financiero, que tiene en el centro no al hombre, sino el dinero,

\footnotetext{
3 Se podría hipotetizar, que este doble movimiento es el mismo que está presente en la labor de "el «ser» y el «hacer» del laico en la Iglesia, quien, por su bautismo y su confirmación, es discípulo y misionero de Jesucristo" (Documento de Aparecida $\mathrm{N}^{\circ}$ 213). Por lo demás, el papa Francisco, incorpora en sus textos al mundo universitario como veremos- la expresión "discipulado misionero".
} 
el dios dinero; en el desarrollo y en el peso de los medios de información, con toda su positividad de comunicación (...). ésta no es una crisis de «cambio»: es una crisis de «cambio de época». Es una época, la que cambia. No son cambios de época superficiales. (Francisco, 2013a) ${ }^{4}$

Para el papa Francisco, esta realidad desafiante marcada por las injusticias y desigualdades tiene un nombre, una "cultura del descarte".

Hay algo que es claro, no podemos seguir dándole la espalda a nuestra realidad, a nuestros hermanos, a nuestra madre la tierra. No nos es lícito ignorar lo que está sucediendo a nuestro alrededor como si determinadas situaciones no existiesen o no tuvieran nada que ver con nuestra realidad. No nos es lícito, más aún no es humano entrar en el juego de la cultura del descarte. (Francisco, 2015c).

... la difusión de la cultura del descarte, que devora cosas y seres humanos sin distinción alguna. Dicho poder se funda en una antropología que concibe al hombre como un depredador y al mundo en el que vive como un recurso para depredar a voluntad. (Francisco, 2018d)

En los escritos del papa Francisco son parte de esta cultura, aspectos tales como una pérdida del "nosotros" fruto de un individualismo que prácticamente tiene sumida a la humanidad en una "guerra mundial en pedazos".

Sin el «nosotros» de un pueblo, de una familia, de una nación y, al mismo tiempo, sin el nosotros del futuro, de los hijos y del mañana; sin el nosotros de una ciudad que «me» trascienda y sea más rica que los intereses individuales, la vida será no sólo cada vez más fracturada sino más conflictiva y violenta. (Francisco, 2018b)

... estamos viviendo en una guerra mundial en pedazos: hay conflictos en muchas regiones del planeta, que ponen en peligro el futuro de generaciones enteras. (Francisco, 2017b)

4 Cada eje temático con sus distintos componentes es acompañado, como máximo, por una, dos o tres frases textuales de los escritos del papa Francisco, pudiendo en muchos casos haberse generado una larga lista de frases con ideas similares. Se ha optado por colocar las frases en un espacio destacado y con identificación del texto, para la facilidad de lectura. De aquí también, que los enunciados del autor que articulan el texto son breves, para facilitar la lectura en forma directa lo indicado por el papa Francisco. 
No obstante, esta dramática realidad que describe el papa Francisco, él mismo señala que frente a esta situación no se puede tener una actitud de pesimismo, ni menos adoptar una actitud apocalíptica.

Cualquier cambio, incluso el actual, es un pasaje que trae consigo dificultades, penurias y sufrimientos, pero también nuevos horizontes para el bien. (Francisco, 2017b)

Nosotros tenemos la tentación de pensar en clave apocalíptica. Esta concepción pesimista de la libertad humana y de los procesos históricos lleva a una especie de parálisis de la inteligencia y de la voluntad (...). Esta es la tentación que nosotros tenemos delante, si vamos por este camino de la desilusión o de la decepción. (Francisco, 2013a)

\section{Una realidad que es superable}

Un segundo eje temático es el llamado a superar la realidad de injusticia y desigualdad y este llamado, coincidente con lo anteriormente indicado, parte siempre de un discurso a no perder la esperanza.

Ante esta dramática realidad os preguntáis, con razón, cuál debería ser nuestra respuesta. Desde luego, no una actitud de desánimo y desconfianza. En particular vosotros, los jóvenes. (Francisco, 2017b)

¡Qué hermoso sería que las aulas universitarias fueran canteras de esperanza, talleres donde se trabaja para un futuro mejor, donde se aprende a ser responsable de uno mismo y del mundo! (Francisco, 2017d)

Detrás del discurso de la esperanza, en los textos del papa Francisco hay siempre una apelación a la confianza en Dios:

Dios nunca decepciona (...). Esto nos da seguridad y gran confianza: una confianza que se apoya en Dios y solicita nuestra colaboración activa y valiente, ante los desafíos del momento presente. (Francisco, 2013b)

La esperanza y la confianza en Dios, a la que invita el papa Francisco, van siempre acompañada de una tarea, construir la "cultura del encuentro".

El aislamiento y la cerrazón en uno mismo o en los propios intereses jamás son el camino para devolver esperanza y obrar una renovación, sino que es la cercanía, la cultura del encuentro (...) la promoción de una cultura del encuentro y del diálogo, orientan hacia la solidaridad, como elemento fundamental para una renovación de nuestras sociedades. (Francisco, 2013a) 
Nuevamente aquí en el papa Francisco hace una apelación a Dios. Salir al encuentro, es encontrase con los demás, es la posibilidad de encontrarse con el rostro de Dios vivo.

Sólo saliendo se encuentra el rostro concreto de los hermanos y hermanas, con sus heridas y sus aspiraciones, sus preguntas y sus dones. Debemos aprender con el corazón, la mente y las manos a «salir del campamento» como dice la Carta a los hebreos $(13,13)$ - para encontrar, precisamente allá afuera, el rostro de Dios en el rostro de cada hermano y hermana. (Francisco, 2019e)

Es la palabra de Dios la que da confianza para enfrentar los desafíos de la realidad y es esa misma palabra de Dios, la que impulsa salir al encuentro: es la tarea encomendada al cristiano.

Ésta es, pues, la tarea que nos ha sido encomendada: encarnar la Palabra de Dios para la Iglesia y para la humanidad del tercer milenio (...), sin tener miedo de arriesgar y de soñar con la paz para todas las personas y todas las naciones. (Francisco, 2018e)

El encuentro -el salir hacia los demás- para el papa Francisco, es el camino para posibilitar un acuerdo que sea base de una nueva civilización. De aquí también, se puede entender, la iniciativa del Santo Padre Francisco para el lanzamiento de un "nuevo pacto educativo".

El pacto es la piedra angular de la creación y de la historia, como nos enseña la Palabra de Dios (...), es una tarea prioritaria educar a los hombres para que vivan este pacto, más aún, para que sean pacto vivo en todas estas dimensiones: para abrir los caminos del futuro a una nueva civilización que abrace en la fraternidad universal a la humanidad y al cosmos. (Francisco, 2019e)

... toda universidad está llamada a ser una «comunidad de estudio, de investigación y de formación» (Constitución Apostólica Veritatis gaudium art. $11 \int 1$ ). Este movimiento de equipo ha estado en crisis desde hace tiempo por varias razones. Por eso, sentí la necesidad de promover el próximo 14 de mayo el día del pacto educativo global. (Francisco, 2020b)

\section{La misión de la universidad}

Un tercer eje temático que se encuentra al analizar los textos del papa Francisco, corresponde a la misión de la universidad. Si bien, cuando el papa Francisco enuncia el rol de una universidad, lo remite a lo que ya 
ha definido Ex Corde Ecclesiae (1995: N³0): "La misión fundamental de la Universidad es la constante búsqueda de la verdad mediante la investigación, la conservación y la comunicación del saber para el bien de la sociedad"; pero, él releva con mucho énfasis, el compromiso de la universidad con la sociedad y en particular con los más vulnerados.

Hay una realidad, un llamado y un conjunto de tareas que el mundo universitario debe realizar, para contribuir a la superación de la injusticia y desigualdad. Su llamado al mundo universitario es incluso un llamado a convertir a la universidad en conciencia crítica.

... no basta con realizar análisis, descripciones de la realidad; es necesario generar los ámbitos, espacios de verdadera búsqueda, debates que generen alternativas a las problemáticas existentes, sobre todo hoy. Que es necesario ir a lo concreto. (Francisco, 2015c)

El ámbito de la promoción social ve a la universidad como una institución que se hace cargo de la sociedad en la que está operando, ejercitando ante todo un papel de conciencia crítica respecto a las diversas formas de poder político, económico y cultural. (Francisco, 2017g)

El papa Francisco indica que, para poder experimentar esta consciencia, es fundamental mantener la autonomía, algo complejo en la sociedad actual; para ello, se requiere de valentía para decir la verdad, libertad de espíritu y honestidad intelectual:

... una universidad hoy en día debe preguntarse siempre si está en peligro de verse limitada por influencias de diversa índole en la libertad de investigación y enseñanza. La presión de poder e intereses, la cuestión de la utilidad, las subvenciones gestionadas y la correspondiente asignación de fondos amenazan con convertirse en criterios finales. (Francisco, 2015b)

... en efecto, una comunidad académica compuesta por profesores y estudiantes de todo el mundo es un lugar adecuado para interpretar los importantes retos de las culturas actuales (...), no puede sino favorecer este proyecto, para que se caracterice por la valentía de la verdad, la libertad de espíritu y la honestidad intelectual. (Francisco, 2020c)

A este desafío de un compromiso de la universidad con la sociedad, de ser conciencia crítica, le agrega el papa Francisco, el compromiso de ser un espacio que vivencia en su interior la sociedad nueva que promulga, siendo un verdadero laboratorio social. 
Podríamos decir que la Universidad se vuelve un laboratorio para el futuro del país, ya que logra incorporar en su seno la vida y el caminar del pueblo superando toda lógica antagónica y elitista del saber. (Francisco, 2018b)

Las universidades, por su naturaleza, están llamadas a ser laboratorios de diálogo y de encuentro al servicio de la verdad, de la justicia y de la defensa de la dignidad humana en todos los niveles. (Francisco 2018c)

Es en este laboratorio donde se debería cultivar con especial dedicación el encuentro y la superación de la fragmentación (dos males de la sociedad actual). Dos tareas por lo demás, que están en la esencia misma de la universidad, ser una comunidad de académicos y estudiantes que se respetan en su diversidad y ser también, un espacio de superación de los límites de las disciplinas particulares.

... un «campo» de crecimiento espiritual y humano, donde a través de una verdadera comunidad de maestros y estudiantes y un intercambio fructífero entre personas de diferentes países y culturas, se hace posible la formación de la mente, así como la formación del corazón. (Francisco, 2016)

En vista de la interdependencia de las grandes cuestiones del futuro, se necesita más que nunca una interdisciplinariedad ejercida con sabiduría y creatividad. En un diálogo estrecho y una auténtica convivencia de disciplinas, surge una síntesis de conocimiento, que puede responder de manera más integral a los desafíos actuales y proporcionar orientación. (Francisco, 2019d)

Para el papa Francisco, esta tarea de encuentro y superación de la fragmentación, es oportunidad también de superar las brechas entre la técnica, las ciencias y el humanismo.

La universidad está llamada a educar a una cultura de la sabiduría, capaz de armonizar el enfoque técnico y científico con el humanista, con la convicción de que la búsqueda de la verdad y la bondad es, en última instancia, única. (Francisco, 2017e)

Hay en los textos del Papa, en esta perspectiva de la universidad como laboratorio del futuro, un intento de mover las fronteras del quehacer universitario. La universidad es en su esencia una comunidad, pero su responsabilidad y compromiso, es con la comunidad amplia de la nación donde se ubica e incluso con la humanidad toda.

... el término «universidad» en sí mismo designa una comunidad, pero también una idea de convergencia de saberes, en una búsqueda que aporte 
verdad y sentido al diálogo entre todos los hombres y mujeres del mundo. (Francisco, 2019f)

Si es este el discurso a las universidades en general, para el papa Francisco esta misión de compromiso con la realidad, se acrecienta en una Universidad Católica.

Es preciso que las instituciones académicas católicas no se aíslen del mundo, sino que entren con valentía en el areópago de las culturas actuales y dialoguen, conscientes del don que tienen para ofrecer a todos. (Francisco, 2014b)

... un «discipulado misionero» se debería percibir de un modo especial en las universidades católicas, que, por su naturaleza misma, están comprometidas en mostrar la armonía entre fe y razón y poner en evidencia la relevancia del mensaje cristiano para una existencia humana vivida en plenitud y autenticidad. (Francisco, 2014a)

... habrá logrado sus objetivos si puede llevar al tejido social esas dosis de profesionalidad y humanidad, que son propias del cristiano que ha sabido buscar con pasión esa síntesis entre la fe y la razón. (Francisco, 2017c)

En este mismo marco, de exigencia a las universidades católicas, el papa Francisco, menciona exigencias fuertes en específico a las Facultades de Teología en particular

No se conformen con una teología de despacho. Que el lugar de sus reflexiones sean las fronteras. Y no caigan en la tentación de pintarlas, perfumarlas, acomodarlas un poco y domesticarlas. También los buenos teólogos, como los buenos pastores, huelen a pueblo y a calle. (Francisco, 2015a)

El papa Francisco insiste, que la fe es un Don que amplía los horizontes universitarios y posibilita una visión más integral. Lo contrario, indica en más de una ocasión, es ver al hombre solo como "material humano".

Permitidme deciros: no tengáis temor a abriros también a los horizontes de la trascendencia, al encuentro con Cristo y a profundizar en la relación con Él. La fe no reduce jamás el espacio de la razón, sino que lo abre a una visión integral del hombre y de la realidad, y defiende del peligro de reducir el hombre a «material humano». (Francisco, 2013a) 
En este marco de una universidad católica animada por la fe que se compromete profundamente con hacer de la realidad un mejor lugar para la humanidad, el papa Francisco le habla a la comunidad universitaria en general y a los estudiantes en forma especial. A la comunidad la llama a formar personas integras, repitiendo numerosas veces el discurso de la educación de la cabeza, el corazón y las manos.

Educar, en general, pero sobre todo en las universidades, no es sólo llenar la cabeza de conceptos. Se necesitan los tres lenguajes. Es necesario que entren en juego los tres lenguajes: el lenguaje de la mente, el lenguaje del corazón y el lenguaje de las manos, para que se piense en armonía con lo que se siente y se hace; se sienta en armonía con lo que se piensa y se hace, se haga en armonía con lo que se siente y se piensa. (Francisco, 2019c)

Pero también hace un llamado a interrogarse por el sentido del trabajo educativo de la universidad: ¿se están formando sus estudiantes para el servicio?

Ningún estudiante de esta universidad debería graduarse sin haber aprendido cómo elegir, responsable y libremente, lo que en conciencia sabe que es lo mejor. Que, en cada situación, incluso en las más complejas, se interesen por lo que en su conducta es justo y humano, cabal y responsable, decididos defensores de los vulnerables. (Francisco, 2019g)

Es justo que nos interroguemos: ¿Cómo ayudamos a nuestros alumnos a no mirar un grado universitario como sinónimo de mayor posición, sinónimo de más dinero o mayor prestigio social? No son sinónimos. (Francisco, 2017f)

Coincidente con la convocatoria que hace a las universidades, el papa Francisco llama a los estudiantes a colocar su formación al servicio de los demás, a ser protagonistas de una sociedad más justa que aquella que reciben en sus manos.

El estudio universitario de calidad, más que ser considerado el privilegio de unos pocos, tiene que ir acompañado por la conciencia de saberse servidores de la justicia y del bien común; servicio a implementarse en el área que a cada uno le toque desarrollar. (Francisco, 2019g)

Los estudios que hagas en esta Universidad serán fructíferos y útiles sólo en la medida en que no os desaconsejarán de esta pertenencia consciente a la historia del pueblo y de la humanidad en su conjunto. (Francisco, 2019b) 
Nos alienta la creencia de que las nuevas generaciones, educadas cristianamente en el diálogo, saldrán de las aulas universitarias motivados para construir puentes y, por lo tanto, para encontrar nuevas respuestas a los muchos desafíos de nuestro tiempo. (Francisco, 2017a)

A esta tarea que llama el papa Francisco a la universidad católica y a sus estudiantes en particular, no es otra que la que él solicita a toda la iglesia y en ello a las universidades católicas como parte de esta: "salir".

La Universidad es una frontera que os espera, una periferia en la que hay que acoger y aliviar las pobrezas existenciales del hombre. (Francisco, 2014d)

... ciertamente no significa alterar el sentido institucional y las tradiciones consolidadas de nuestras realidades académicas, sino más bien orientar su función en la perspectiva de una Iglesia más marcadamente «en salida»y misionera (...). Ésta es, pues, la tarea que nos ha sido encomendada: encarnar la Palabra de Dios para la Iglesia y para la humanidad del tercer milenio. (Francisco, 2018e)

\section{CONCLUSIONES}

Haciendo dialogar la introducción a este artículo y lo encontrado en el análisis de los textos del papa Francisco sobre la universidad, es posible apreciar que en sus discursos, cartas y homilías, parte de los grandes temas que desafían a la universidad actual, no son desarrollados e incluso algunos no están presentes; pero lo que si se encuentra en el análisis de los textos del papa Francisco, coincidentemente tanto en el conteo de palabras más utilizadas como en el análisis temático, que es posible identificar tres grandes temas que articulan sus discursos con relación al mundo universitario. Utilizando expresiones del mismo Papa: una realidad desafiante caracterizada como una "cultura del descarte"; la urgencia de instalar una "cultura del encuentro" como respuesta a la realidad que apremia y la necesidad de una "universidad en salida", que trasciende sus fronteras para ir -desde su especificad académica- a enfrentar los males que sufre la humanidad.

Dentro del primer eje temático de diagnóstico de la realidad, se articulan a su alrededor temas como la realidad social, la situación del mundo, lo que se vive actualmente y acontece hoy. En su diagnóstico, la sociedad actual es una sociedad en cambio, de incertidumbre, de individualismo, de consumismo, de destrucción de la tierra, de la pérdida de un "nosotros" que permita la necesaria confianza y cohesión social y ello, está llevando a una verdadera "guerra mundial en pedazos". Lo que el 
papa Francisco sintetiza con la expresión: "cultura del descarte". Una cultura que ya no busca "integrar", como dice Jesús Martín-Barbero (2004), aunque sea para explotar, sino que excluye a una parte importante de la población.

No obstante, esta dramática realidad, el papa Francisco señala que esta es una oportunidad que puede superarse -para bien- si se enfrenta con esperanza. De aquí el segundo eje temático de su discurso, que se puede mejorar la realidad confiando en Dios y trabajando en la construcción de una "cultura del encuentro". Para el papa Francisco salir al encuentro, es salir a encontrase con los más pobres y en ello encontrarse con el rostro de Dios. Salir al encuentro es un acto y un deber de fraternidad, es asumir que la humanidad toda es hijo del mismo Padre Dios. Salir hacia los demás para el papa Francisco, además, es el camino para posibilitar el pacto social necesario para una nueva civilización.

El tercer eje temático que se encuentra al hacer un análisis de los textos del papa Francisco, corresponde a la misión de la universidad. En este caso, sin dejar de reafirmar lo expresado en Ex Corde Ecclesiae, hay en él, un importante énfasis sobre el compromiso de la universidad con la sociedad y en particular con los más vulnerados dentro de ella. En este sentido, el Papa llama a extender las fronteras de responsabilidad de la comunidad universitaria más allá de sus límites, asumiendo como suyas las urgencias de la humanidad. Las que deben estar presente en los currículos y en las mesas de trabajo de los investigadores.

Hay una realidad que urge una "cultura del descarte" y una tarea a hacer, instalar "una cultura del encuentro" y es a ello, lo que está llamado a contribuir significativamente el mundo universitario. La universidad debe convertirse en conciencia crítica, pero también, un laboratorio donde se cultive el encuentro y la superación de la fragmentación. Dos tareas que están en la esencia misma de la universidad: ser una comunidad donde se dialoga y se respetan la diversidad y ser espacio de superación de los límites de las disciplinas particulares, en especial la brecha entre la técnica y el humanismo. Ser conciencia y laboratorio de una nueva sociedad, indica el papa Francisco no es una tarea fácil, dado lo complejo que es mantener la autonomía necesaria; pero, además, porque se requiere de valentía para decir la verdad, libertad de espíritu y honestidad intelectual.

$\mathrm{Si}$ es este el discurso a las universidades en general, para el papa Francisco esta misión de compromiso con la realidad, se acrecienta en una Universidad Católica. Ya que la fe es un Don que amplía los horizontes universitarios y posibilita una visión más integral; lo contrario, indica, es ver al hombre solo como "material humano". En este marco convoca a la universidad católica a formar personas integras, mediante la educación en conjunto de la cabeza, el corazón y las manos y la invita, 
además, a interrogarse si el proceso educativo está efectivamente formando para el compromiso con los demás. A los estudiantes, a su vez, el papa Francisco les insiste en colocar su formación al servicio de los más pobres y ser protagonistas de una sociedad más justa que aquella que reciben en sus manos. En síntesis, el papa Francisco, convoca a las universidades católicas y a sus estudiantes en particular, a lo que él solicita a toda la iglesia, que las universidades se conviertan en "universidades en salida".

Al momento de inferir más allá de lo que está explícito en los textos analizado, hay un primer aspecto no siempre visible que está presente en los discursos del papa Francisco al mundo universitario. Si bien él insiste en plantear que: "No quiero dar una lección académica, aunque el contexto y vosotros que sois un grupo cualificado tal vez lo requerirían. Prefiero ofrecer algunas reflexiones en voz alta que parten de mi experiencia de hombre y de Pastor de la Iglesia" (Francisco, 2013a). No obstante, lo recién indicado, su diagnóstico sobre la realidad mundial y sus propuestas al respecto, poseen muchas veces referencias -en algunos casos no explicitadas en el discurso, pero si como pie de página o bibliografía- a autores contemporáneos con los que coinciden sus apreciaciones. Un buen ejemplo de ello es el texto del discurso en la Pontificia Universidad Católica de Chile (2018) donde indica que: "En esta sociedad líquida o ligera, como la han querido denominar algunos pensadores, van desapareciendo los puntos de referencia desde donde las personas pueden construirse individual y socialmente". Solo al consultar el texto escrito son explicitas las referencias a Bauman y Lipovetsky.

Es Bauman (2004) quien identifica a la sociedad de hoy como una sociedad líquida, una sociedad de transformación, de cambio permanente y es Lipovetsky, a su vez (2016: 25), quien caracteriza a la sociedad actual como de ligereza. "Lo ligero nutre cada vez más nuestro mundo material y cultural, ha invadido nuestras prácticas cotidianas y remodelado nuestro imaginario (...) ninguna idea aclara mejor la dinámica de las sociedades modernas que la de «aligeramiento de la vida»".

Un segundo aspecto, incluso más hipotético que el anterior, es el profundo apego a Ex Corde Ecclesiae, lo que desde luego no resulta una sorpresa, pero en lo que llama la atención, es que pareciera ser que su plan de trabajo con relación al mundo universitario, no fuera otro que recordar un punto en particular de esta Constitución Apostólica (1995: $\mathrm{n}^{\circ} 32$ ). Aquel que hace referencia a las tareas de una Universidad Católica: "el estudio de los graves problemas contemporáneos, tales como, la dignidad de la vida humana, la promoción de la justicia para todos, la calidad de vida personal y familiar, la protección de la naturaleza, la búsqueda de la paz y de la estabilidad política, una distribución más equitati- 
va de los recursos del mundo y un nuevo ordenamiento económico y político".

Hay en su diagnóstico una realidad de profundos y vertiginosos cambios, que está trayendo injusticias y desigualdades, que urge de respuestas nuevas; estamos viviendo una sociedad que se ha vuelto liquida $o$ ligera (como ya se mencionó) donde el individualismo y el consumismo se señorean, y todo ello, ha ido haciendo perder el sentido de las cosas. En el Documento de Aparecida (2007: $n^{\circ} 37$ ), que conoce muy bien el papa Francisco, se plantea que "muchos estudiosos de nuestra época han sostenido que la realidad ha traído aparejada una crisis de sentido. Ellos no se refieren a los múltiples sentidos parciales que cada uno puede encontrar en las acciones cotidianas que realiza, sino al sentido que da unidad a todo lo que existe". Quizás por ello, el llamado más reciente del papa Francisco al mundo universitario, es a abrir "las puertas de la Universidad del Sentido. Porque educar es buscar el sentido de las cosas. Es enseñar a buscar el sentido de las cosas" (Francisco, 2020a).

Pareciera, en síntesis, que los escritos del papa Francisco, en una mirada simple, optan por no centrase tanto en el "ser" de la Universidad, sino más bien en el "hacer de ella"; pero, a diferencia de esta lectura simple, detrás del discurso del papa Francisco, hay énfasis en un hacer que reconfigura el ser mismo de la universidad, incorporando lo que muchos denominan la "tercera misión de la universidad". Si bien en América Latina, la docencia y la investigación, han estado por décadas unidas primero a la "extensión" y hoy a la "vinculación con el medio"; ello es un fenómeno novedoso en el mundo europeo, que en forma posterior a la promulgación de Ex Corde Ecclesiae (1990), inicia la tarea de definir esta "tercera misión". Bueno (2007), indica al respecto que: "En la última década del siglo XX se ha iniciado un movimiento de revisión sobre la misión de la universidad en la actual sociedad del conocimiento, incorporando a sus funciones tradicionales de enseñanza superior y de investigación una tercera".

En los desafíos que debe enfrentar la universidad en su conjunto y cada universitario en particular: su responsabilidad con su nación y la humanidad toda; el papa Francisco, reconceptualiza muchos de los conceptos centrales del ser mismo de la universidad. La comunidad universitaria no encuentra sus límites en la academia, sino que se extiende a la

5 Muchos historiadores sobre universidades, como Tünnermann (2008), vinculan la tarea de extensión con el movimiento universitario de Córdoba (1918), Argentina. En la actualidad, esta misión que se origina como un servicio de la academia a quienes no están dentro de ella, modificó su visión unilateral de quien sabe y extiende su creación cultural a los demás, a una perspectiva bilateral, donde se enseña, pero también se aprende de los de afuera. 
sociedad toda; el diálogo de saberes no se restringe a las disciplinas que habitan los campus universitarios, sino que incorpora los saberes populares que se originan fuera de estos espacios; como tampoco, la formación universitaria de calidad se mide solo por lo que produce, sino también, por los servicios que presta y a quiénes los presta. Un buen resumen de esta reconceptualización, en las palabras del propio papa Francisco, es su planteamiento de que la comunidad universitaria, no puede "construir conocimiento al margen de los destinatarios de los mismos. Es necesario que la adquisición de conocimiento sepa generar una interacción entre el aula y la sabiduría de los pueblos" (Francisco, 2018b).

\section{REFERENCIAS}

Barber, M., Donnelly, K. \& Rizvi, S. (2013). An avances coming. Higher education and the revolution ahead. London: Institute for Public Policy Research.

Bauman, Z. (2004). Modernidad líquida. Buenos Aires: Fondo de Cultura Económica.

Braun, V. \& Clarke, V. (2006). Using thematic analysis in psychology. Qualitative Research in Psychology, 3(2), 77-101.

Bueno Campos, E. (2007). La tercera misión de la universidad: El reto de la transferencia del conocimiento. Revista de Investigación en Gestión de la Innovación $y$ Tecnología, (41). Disponible en http://www.madrimasd.org/revista/revista41/tribuna/tribuna2.asp

Cuartas, C. (2003). La idea de universidad en Juan Pablo II. Revista Theologica Xaveriana, (146), 163-190.

Francisco (2013a). Encuentro con el mundo de la Cultura. Pontificia Facultad de Teología de Cerdeña. Disponible en http:/ /www.vatican.va/content/francesco/es/speeches/2013/september/ documents/papa-francesco_20130922_cultura-cagliari.html

Francisco (2013b). Celebración de las Vísperas con la participación de los universitarios romanos. Disponible en http://www.vatican.va/content/francesco/es/homilies/2013/documents /papa-francesco_20131130_vespri-universitari-romani.html

Francisco (2014a). Discurso a delegación de la Universidad de "Notre Dame", Indiana, EE.UU. Disponible en https://www.vatican.va/content/francesco/es/speeches/2014/january/d ocuments/papa-francesco_20140130_university-notre-dame.html

Francisco (2014b). Discurso a participantes Plenaria de la Congregación para la Educación católica. Disponible en http://www.vatican.va/content/francesco/es/speeches/2014/february/d ocuments/papa-francesco_20140213_congregazione-educazionecattolica.html

Francisco (2014c). Discurso a la comunidad de la Pontificia Universidad Gregoriana y a los miembros de los asociados pontificios Instituto Bíblico y 
Pontificio Instituto Oriental; Aula Pablo VI. Disponible en http://www.vatican.va/content/francesco/es/speeches/2014/april/docu ments/papa-francesco_20140410_universita-consortiumgregorianum.html

Francisco (2014d). Mensaje a la Federación Universitaria Católica Italiana. Disponible en http://www.vatican.va/content/francesco/es/messages/pontmessages/2014/documents/papa-francesco_20141014_messaggiofuci.html

Francisco (2015a). Carta al Gran Canciller de la Pontificia Universidad Católica Argentina en el Centenario de la Facultad de Teología. Vaticano. Disponible https://www.vatican.va/content/francesco/es/letters/2015/documents/ papa-francesco_20150303_lettera-universita-cattolica-argentina.html

Francisco (2015b). Botschaft zur 650-Jahar-Feier der Universität Wien. Aus dem Vatikan. Disponible en https://www.vatican.va/content/francesco/de/messages/pontmessages/2015/documents/papa-francesco_20150312_messaggiouniversita-vienna.html

Francisco (2015c). Encuentro con el mundo de la enseñanza. Pontificia Universidad Católica de Ecuador. Disponible en http://www.vatican.va/content/francesco/es/speeches/2015/july/docu ments/papa-francesco_20150707_ecuador-scuola-universita.html

Francisco (2016). Schreiben zum 90-Jährigen Bestehen der PhilosophischTheologischen Hochschule Sant Georgen. Vatikan. Disponible en http://www.vatican.va/content/francesco/de/letters/2016/documents/p apa-francesco_20161004_giubileo-sankt-georgen.html

Francisco (2017a). Discorso ai participanti alla Plenaria della Congregazione per L'Educazione Cattolica -Degli Instituti di Studi- Sala Clementina. Disponible http://www.vatican.va/content/francesco/it/speeches/2017/february/do cuments/papa-francesco_20170209_plenaria-educazione-cattolica.html

Francisco (2017b). Discurso en la Universidad Roma Tre. Disponible en http://www.vatican.va/content/francesco/es/speeches/2017/february/d ocuments/papa-francesco_20170217_universita-romatre.html

Francisco (2017c). Carta al Emmo. Cardenal Giuseppe Versaldi, Gran Canciller de la Pontificia Universidad Católica del Perú con motivo del primer centenario del Ateneo. Disponible en https://press.vatican.va/content/salastampa/es/bollettino/pubblico/2017 /04/01/car.html

Francisco (2017d). Encuentro con los estudiantes y el mundo académico. Bolonia. cuments/papa-francesco_20171001_visitapastorale-bolognamondoaccademico.html 
Francisco (2017e). Saludo a una delegación de la Universidad de Tel Aviv. Sala de los Papas. Disponible en http://www.vatican.va/content/francesco/es/speeches/2017/october/do cuments/papa-francesco_20171023_delegazione-telaviv-university.html

Francisco (2017f). Discurso a la comunidad de la Universidad Católica Portuguesa con ocasión del 50 Aniversario de su Fundación. Sala Clementina. Disponible

http://www.vatican.va/content/francesco/es/speeches/2017/october/do cuments/papa-francesco_20171026_universita-cattolica-portoghese.html

Francisco (2017g). Discurso a los miembros de la Federación Internacional de las Universidades Católicas. Disponible en http://www.vatican.va/content/francesco/es/speeches/2017/november/ documents/papa-francesco_20171104_federazione-universitacattoliche.html

Francisco (2018a). Exhortación Apostólica Gaudete et Exsultate. Disponible en http://www.vatican.va/content/francesco/es/apost_exhortations/docum ents/papa-francesco_esortazione-ap_20180319_gaudete-et-exsultate.html

Francisco (2018b). Discurso: Visita a la Pontificia Universidad Católica de Chile.

https://www.vatican.va/content/francesco/es/speeches/2018/january/d ocuments/papa-francesco_20180117_cile-santiago-pontuniversita.html

Francisco (2018c). Discurso a una delegación de la Villanova University de Filadelfia.

Disponible

en http://www.vatican.va/content/francesco/es/speeches/2018/april/docu ments/papa-francesco_20180414_universita-villanova.html

Francisco (2018d). Discurso a los miembros de la Fundación "Gravissimum Educationis". http://www.vatican.va/content/francesco/es/speeches/2018/june/docu ments/papa-francesco_20180625_gravissimum-educationis.html

Francisco (2018e). Carta al Cardenal Angelo de Donatis con motivo de la Inauguración del Año Académico de la Universidad Lateranense y de la institución de un nuevo curso de estudios en "Ciencias de la Paz". Disponible en http://www.vatican.va/content/francesco/es/letters/2018/documents/p apa-francesco_20181112_lettera-cardinale-dedonatis.html

Francisco (2019a). Mensaje para el lanzamiento del Pacto Educativo. Vaticano, 12 de septiembre. Disponible en http://www.vatican.va/content/francesco/es/messages/pontmessages/2019/documents/papa-francesco_20190912_messaggio-pattoeducativo.html

Francisco (2019b). Lectio Divina: Pontificia Università Lateranense. Disponible en

http://www.vatican.va/content/francesco/it/speeches/2019/march/doc uments/papa-francesco_20190326_lectiodivina-univlateranense.html

Francisco (2019c). Discurso a los participantes en el Foro Anual "Nuevas fronteras para líderes universitarios" de la Federación Internacional de Univer- 
sidades Católicas. Disponible en http://www.vatican.va/content/francesco/es/speeches/2019/november/ documents/papa-francesco_20191104_dirigenti-universita.html

Francisco (2019d). Botschaft zum Gründungsjubiläum der Universität Rostock.

Disponible

en

http://www.vatican.va/content/francesco/de/messages/pontmessages/2019/documents/papa-francesco_20191112_giubileofondazione-universita-rostock.html

Francisco (2019e). Discurso a la Comunidad Académica del Instituto Universitario Sophia de Loppiano. Disponible en http://www.vatican.va/content/francesco/es/speeches/2019/november/ documents/papa-francesco_20191114_loppiano-univ-sophia.html

Francisco (2019f). Discurso a los Profesores y Alumnos de la Libre Universidad María Asunta LUMSA. Disponible en http://www.vatican.va/content/francesco/es/speeches/2019/november/ documents/papa-francesco_20191114_lumsa.html

Francisco (2019g). Discurso: Visita a la Universidad Sofía de Tokio. Disponible en

http://www.vatican.va/content/francesco/es/speeches/2019/november/ documents/papa-francesco_20191126_universita-tokyo.html

Francisco (2020a). Video mensaje del Santo Padre Francisco con ocasión del ciber-encuentro mundial organizado por la Fundación Scholas occurrentes. Disponible en https://www.vatican.va/content/francesco/es/messages/pontmessages/2020/documents/papa-francesco_20200605_videomessaggioscholas-occurrentes.html

Francisco (2020b). Discurso a los participantes en la Asamblea Plenaria de la Congregación para la Educación Católica de los Institutos de Estudios. Sala Clementina. Disponible en http://www.vatican.va/content/francesco/es/speeches/2020/february/d ocuments/papa-francesco_20200220_congregaz-educaz-cattolica.html

Francisco (2020c). Carta al Rector de la Universidad Pontificia Santo Tomás de Aquino.

Disponible en http://www.vatican.va/content/francesco/es/letters/2020/documents/p apa-francesco_20200518_istituto-cultura-gp2-angelicum.html

Francisco (2020d). Video mensaje con ocasión del ciberencuentro mundial organizado por la Fundación Scholas Occurrentes. Disponible en http://www.vatican.va/content/francesco/es/messages/pontmessages/2020/documents/papa-francesco_20200605_videomessaggioscholas-occurrentes.html

Global University Network for Innovation (2019). Higher Education in the World 7. Higher Education in the World: Synergies between Science, Technology and Humanities. Disponible en http://www.guninetwork.org/files/download_full_report_heiw7.pdf 
Juan Pablo II (1990). Constitución Apostólica Ex Corde Ecclesiae. Sobre las Universidades Católicas. Disponible en http://www.vatican.va/content/johnpaul-ii/es/apost_constitutions/documents/hf_jp-ii_apc_15081990_excorde-ecclesiae.html

Lipovetsky, G. (2016) De la ligereza. Barcelona: Anagrama.

Martín - Barbero, J. (2004): Crisis identitaria y transformaciones de la subjetividad. En $\mathrm{M}^{\mathrm{a}} \mathrm{C}$. Laverde et al. (Eds.), Debates sobre el sujeto. Perspectivas contemporáneas. (pp. 33-45). Bogotá: Ed. Universidad Central DIUC - Siglo del Hombre Editores.

Páramo, Má Á. (2010). Análisis cualitativo de discursos grupales asistido por programa de software TextSTAT. Valoración de su utilidad en la exploración y relación de significados. Revista Liberabit, 16(2), 141-151.

Rodríguez, Ma . J. \& Garrigós, J. I. (2017). Análisis sociológico con documentos personales. Cuadernos de Metodología N 57. Madrid: Centro de Investigaciones Sociológicas (CIS).

Santander, P. (2011). Por qué y cómo hacer análisis de discurso. Revista Cinta Moebio, (41), 207-224.

Tünnermann, C. (2008). Noventa años de la Reforma Universitaria de Córdoba: 1918 2008. Buenos Aires: Edición Consejo Latinoamericano de Ciencias Sociales, CLACSO.

Viñado, F. (2017). La idea de Universidad en el magisterio pontificio de Benedicto XVI. (Tesis Doctoral de la Facultad de Educación, Departamento de Teoría e historia de la educación). Madrid: Universidad Complutense de Madrid.

Zani, A. (2015). Identidad y misión de las Universidades Católicas a la luz de los nuevos tiempos. Congreso de las Universidades Católicas en Pontificia Universidad Católica de Chile, 2 de septiembre de 2015. Disponible en: https://www.youtube.com/watch?v $=y 4 A u A C d X d x k$ 